\title{
Peripheral nervous system injury after high-dose single-fraction image-guided stereotactic radiosurgery for spine tumors
}

\author{
Michael D. Stubblefield, MD, ${ }^{1}$ Katarzyna Ibanez, MD, ${ }^{2}$ Elyn R. Riedel, MA, ${ }^{3}$ Ori Barzilai, MD, ${ }^{4}$ \\ Ilya Laufer, MD, ${ }^{4,7}$ Eric Lis, MD, ${ }^{5}$ Yoshiya Yamada, MD, ${ }^{6}$ and Mark H. Bilsky, MD ${ }^{4,7}$ \\ ${ }^{1}$ Kessler Institute for Rehabilitation Select Medical, West Orange, New Jersey; ${ }^{2}$ Department of Neurology, Rehabilitation \\ Medicine Service; Departments of ${ }^{3}$ Epidemiology and Biostatistics, ${ }^{4}$ Neurosurgery, ${ }^{5}$ Radiology, and ${ }^{6}$ Radiation Oncology, \\ Memorial Sloan Kettering Cancer Center, New York; and 'Department of Neurological Surgery, Weill Cornell Medical College, \\ New York, New York
}

OBJECTIVE The object of this study was to determine the percentage of high-dose (1800-2600 cGy) single-fraction stereotactic radiosurgery (SF-SRS) treatments to the spine that result in peripheral nervous system (PNS) injury.

METHODS All patients treated with SF-SRS for primary or metastatic spine tumors between January 2004 and May 2013 and referred to the Rehabilitation Medicine Service for evaluation and treatment of neuromuscular, musculoskeletal, or functional impairments or pain were retrospectively identified.

RESULTS Five hundred fifty-seven SF-SRS treatments in 447 patients resulted in 14 PNS injuries in 13 patients. All injures resulted from SF-SRS delivered to the cervical or lumbosacral spine at $2400 \mathrm{cGy}$. The overall percentage of SFSRS treatments resulting in PNS injury was $2.5 \%$, increasing to $4.5 \%$ when the thoracic spine was excluded from analysis. The median time to symptom onset following SF-SRS was 10 months (range 4-32 months). The plexus (cervical, brachial, and/or lumbosacral) was affected clinically and/or electrophysiologically in 12 (86\%) of 14 cases, the nerve root in $2(14 \%)$ of 14 , and both in $6(43 \%)$ of 14 cases. All patients experienced pain and most $(93 \%)$ developed weakness. Peripheral nervous system injuries were CTCAE Grade 1 in 14\% of cases, 2 in $64 \%$, and 3 in $21 \%$. No dose relationship between SF-SRS dose and PNS injury was detected.

CONCLUSIONS Single-fraction SRS to the spine can result in PNS injury with major implications for function and quality of life.

https://thejns.org/doi/abs/10.3171/2016.11.FOCUS16348

KEY WORDS spine; radiosurgery; peripheral nerve; complications

$\mathrm{H}$ IGH-DOSE single-fraction stereotactic radiosurgery (SF-SRS) has become a valuable technique for managing primary and metastatic spine tumors. It allows for tightly conformal treatment plans characterized by a steep dose gradient between target volumes and adjacent radiosensitive normal tissues such as the spinal cord, brachial plexus, and esophagus.

Multiple reports have demonstrated the safety and efficacy of SF-SRS for a variety of tumor types with or without tumor separation surgery and/or prior radiation. ${ }^{1,6,9-11,16,22,26,28,31,35,36}$ Local control and progression-free survival rates of approximately $90 \%$ with good palliation and symptomatic improvement in $80 \%-90 \%$ of patients are consistently reported. . $9,11,22,26,28,31,35,36$ Although there have yet to be definitive trials comparing SF-SRS to conventional radiotherapy techniques, SF-SRS does compare favorably to traditional treatment in terms of local control and palliation. ${ }^{19}$

Toxicity related to SF-SRS is not well defined but is generally considered to be low. Reported complications include vertebral body fracture, skin reactions, dyspnea, gastrointestinal dysfunction, esophagitis, tracheoesopha-

ABBREVIATIONS CTCAE = Common Terminology Criteria for Adverse Events; DRG = dorsal root ganglion; EGFR = epidermal growth factor; EMG = electromyography; GTV = gross tumor volume; PNS = peripheral nervous system; PTV = planning treatment volume; SF-SRS = single-fraction stereotactic radiosurgery; VEGF = vascular endothelial growth factor.

SUBMITTED August 22, 2016. ACCEPTED November 14, 2016.

INCLUDE WHEN CITING DOI: 10.3171/2016.11.FOCUS16348. 
geal fistula, odynophagia, and wound issues. $3,6,9,16,28,31,32,35,36$ Myelopathy has been described in several studies, often in association with prior radiation, and with an overall incidence $<1 \% .^{11,12,33,34,36}$ Use of an epidermal growth factor receptor (EGFR) inhibitor within 2 months of radiation treatment has been cited as a factor possibly contributing to the development of myelopathy following SF-SRS. ${ }^{11}$

Reports of peripheral nervous system (PNS) injury occurring in association with SF-SRS are rare. The few described events have usually been transient or associated with tumor involvement of the affected neural structure. ${ }^{6,26,28,36}$ Peripheral nervous system injury has been reported following SF-SRS to other structures. For instance, damage to the optic nerve has been reported at a rate of $5 \%$ of patients treated for craniopharyngioma using SF-SRS at a high-dose $(>1500 \mathrm{cGy})$ as well as a low dose $(<800$ cGy) in the setting of prior irradiation..$^{14}$ A retrospective review of 222 patients with benign skull base tumors has demonstrated that the chance of radiation-induced optic neuropathy following SF-SRS was $1 \%$ for patients receiving $>800$ cGy but increased to $10 \%$ for those treated with $>1200 \mathrm{cGy}^{23}$ A study of SF-SRS versus hypofractionated (HF)-SRS for vestibular schwannoma demonstrated radiation-induced facial nerve damage at a rate of $16 \%$ for SF-SRS (dose range $1100 \mathrm{cGy}$ to $1400 \mathrm{cGy}$ ) and 3\% for HF-SRS (10 fractions of 300-400 cGy or 25 fractions of $200 \mathrm{cGy}) .^{2}$

At our institution, the dose of radiation used in SF-SRS to the spine is between 1800 and 2400 cGy without attempts to restrict the radiation dose to the nerve roots and/ or plexus. While there are studies reporting a relatively high rate of PNS dysfunction following treatment of tumors proximate to nerve structures at the cranial base, few reports of PNS damage following SF-SRS to the spine have been described.

In this retrospective review we describe PNS injury affecting the nerve root and/or plexus (termed "radiculoplexopathy") following SF-SRS in a large cohort of patients with primary and metastatic tumors of the spine.

\section{Methods}

All patients treated with high-dose SF-SRS for primary or metastatic spine disease between January 2004 and May 2013 and referred to the Rehabilitation Medicine Service at the Memorial Sloan Kettering Cancer Center for evaluation and treatment of neuromuscular, musculoskeletal, functional impairments, or pain were identified through a search of a proprietary institutional database. All patients had a histologically confirmed solid tumor and radiographic evidence of a primary or metastatic spinal tumor. The study was performed in compliance with the Health Insurance Portability and Accountability Act (HIPAA) and the standards of the Institutional Committee on Human Experimentation in accordance with the Declaration of Helsinki of 1975. Our request for waiver of the HIPAA authorization and informed consent was granted by the institutional review board.

All patients in our study had undergone spine SF-SRS simulation, planning, and delivery, as previously described. ${ }^{25,35}$ Supine CT myelography was used for simu- lation in most patients. Target volume was defined in accordance with the consensus guidelines published by the International Spine Radiosurgery Consortium. ${ }^{4}$ The planning treatment volume (PTV) was contoured to the thecal sac, and every PTV included the proximal spinal nerve roots for each treated spinal segment. No attempt was made to reduce the dose to the spinal nerve roots. Inverse treatment planning with intensity modulation was performed using in-house software. ${ }^{24,27}$ Treatment plans were normalized to the $100 \%$ isodose line to maximize the percentage of PTV receiving the prescribed dose without exceeding dose limits for normal tissue such as the spinal cord. Treatment was delivered to the $100 \%$ isodose line with 6- and/or 15-MV photons. The spinal cord was limited to a maximal dose of 1200-1400 cGy. All of the exiting nerve roots were included in the PTV for all patients in the series. On-board $2 \mathrm{D} \mathrm{kV}$ and $3 \mathrm{D} \mathrm{kV}$ (cone beam) imaging was used to verify position for each treatment. Cytotoxic systemic chemotherapy was discontinued for 7-10 days before and after SF-SRS.

Patients were evaluated 8 weeks posttreatment and every 3-4 months thereafter. An MRI study of the entire spine with and without gadolinium was usually performed prior to each posttreatment visit. Patients who developed neuromuscular, musculoskeletal, or functional deficits or pain were referred to the Rehabilitation Medicine Service for evaluation and treatment.

All data were retrospectively collected from a review of clinic notes including those from oncology, neurosurgery, radiation oncology, rehabilitation medicine, neurology, and others as appropriate. Radiculopathy and/or plexopathy were graded retrospectively based on chart review in accordance with the severity scale set forth by the National Institutes of Health Common Terminology Criteria for Adverse Events (CTCAE) v4.03. ${ }^{5}$

Electrodiagnostic testing (electromyography [EMG]) was performed in most patients referred to the Rehabilitation Medicine Service. All EMG studies were performed by a physician board-certified in electrodiagnostic medicine (American Board of Electrodiagnostic Medicine or clinical neurophysiology certification) who had considerable experience performing and interpreting EMG in the cancer population.

A Sierra Wave machine (Cadwell Laboratories Inc.) was used at standard settings and with pre-specified normative data for all electrophysiological testing. Normative laboratory values were based on data compiled by an allied institution using identical techniques and machine settings. Determining which specific electrophysiological investigations to perform was based on our laboratory standards and modified as clinically appropriate by the examining physician. Needle EMG was performed either unilaterally or bilaterally with the intent to confirm cervical or lumbosacral radiculopathy and cervical, brachial, or lumbosacral plexopathy and to exclude competing disorders such as peripheral neuropathy and mononeuropathy.

Patient-level and treatment-level characteristics were summarized using descriptive statistics. A cumulative incidence curve estimating the development of nerve injuries was calculated, with death considered as a competing event. ${ }^{18}$ The association of radiation dose (categorized as 
$<2400$ or $\geq 2400$ cGy) with the development of nerve injuries was evaluated using a modified chi-square statistic. ${ }^{13}$ Summary statistics were calculated using SAS 9.4 (SAS Institute Inc.). The cumulative incidence curve was generated, and the competing risks analysis was performed in R 3.1.1 using the CMPRSK package.

\section{Results}

Patient and treatment characteristics are summarized in Tables 1 and 2. A total of 447 patients received 557 treatments with SF-SRS doses ranging from 1800 to 2600 cGy. The majority, 399 patients, received 494 treatments at doses of 2400-2600 cGy. Slightly more subjects were male $(62 \%)$. Ninety-three percent of treated tumors were metastatic, and histologies included carcinoma (71\%), sarcoma (11\%), melanoma (7\%), and other (11\%).

Table 2 details the characteristics of treatments received. Eighty-eight percent of the SF-SRS treatments consisted of a dose of 2400 cGy (median dose 2400 cGy, mean dose $2364 \mathrm{cGy}$ ). The majority of treatments targeted the thoracic spine (52\%), followed by the lumbar spine $(32 \%)$, cervical spine $(12 \%)$, and sacrum $(8 \%)$.

Table 3 summarizes the number and percentage of nerve injuries by the spinal level included in treatment for all patients (1800-2600 cGy) and for those treated with $\geq 2400 \mathrm{cGy}$. A graph of the percentage of nerve injuries by spinal level for all patients is provided in Fig. 1. Fourteen PNS injuries were associated with 557 treatments in 13 patients. One patient suffered 2 different PNS injuries (Injuries 2 and 6) from separate treatments. All 14 PNS injuries occurred when SF-SRS was delivered at 2400 cGy.

A dosimetric analysis of treatment plans by gross tumor volume (GTV) and PTV was performed for patients who did or did not experience neuropathy and, using a Mann-Whitney U-test, was tested for differences between those who did or did not have toxicity. The mean GTV and PTV for patients who experienced neuropathy were 13.2

TABLE 1. Summary of characteristics in 447 patients treated with SF-SRS

\begin{tabular}{cc}
\hline \multicolumn{1}{c}{ Variable } & No. \\
\hline Median age in yrs (range) & $60(16-87)$ \\
\hline Sex & $172(38 \%)$ \\
\hline Female & $275(62 \%)$ \\
\hline Male & \\
\hline Histology & $316(71 \%)$ \\
\hline Carcinoma & $32(7 \%)$ \\
\hline Melanoma & $50(11 \%)$ \\
\hline Sarcoma & $49(11 \%)$ \\
\hline Other & $32(7 \%)$ \\
\hline Tumor stage & $415(93 \%)$ \\
\hline Primary & \\
\hline Metastatic & $98(22 \%)$ \\
\hline Status & $349(78 \%)$ \\
\hline Alive &
\end{tabular}

TABLE 2. Characteristics of 557 treatments for spine tumors

\begin{tabular}{cc}
\hline \multicolumn{1}{c}{ Variable } & Value \\
\hline Spinal region & $66(12 \%)$ \\
\hline Cervical & $292(52 \%)$ \\
\hline Thoracic & $177(32 \%)$ \\
\hline Lumbar & $47(8 \%)$ \\
\hline Sacral & $11(2 \%)$ \\
\hline Prescribed dose (cGy) & $1(<1 \%)$ \\
\hline 1800 & $35(6 \%)$ \\
\hline 2000 & $15(3 \%)$ \\
\hline 2100 & $1(<1 \%)$ \\
\hline 2200 & $491(88 \%)$ \\
\hline 2300 & $1(<1 \%)$ \\
\hline 2400 & $2(<1 \%)$ \\
\hline 2500 & $2400(2400-2400)$ \\
\hline 2600 & $2364(114)$ \\
\hline Median dose in cGy (IQR)
\end{tabular}

$\mathrm{IQR}=$ interquartile range; $\mathrm{SD}=$ standard deviation .

and $60 \mathrm{~cm}^{3}$, respectively. The mean GTV and PTV in 381 available corresponding treatment plans for patients who did not experience neuropathy were 18.4 and $79.9 \mathrm{~cm}^{3}$, respectively. There were no significant differences in lesion size or target volume size between the lesion cases that did and those that did not have neuropathy. Further, there were no significant differences in radiation dose between

TABLE 3. Peripheral nervous system injury by spinal level included in treatment

\begin{tabular}{lccccc}
\hline & \multicolumn{2}{c}{$1800-2600$ cGy } & & \multicolumn{2}{c}{$2400-2600$ cGy } \\
\cline { 2 - 3 } \cline { 5 - 6 } Parameter & $\begin{array}{c}\text { No. of Injuries/ } \\
\text { No. of Spinal } \\
\text { Levels Treated }\end{array}$ & $\%$ & & $\begin{array}{c}\text { No. of Injuries/ } \\
\text { No. of Spinal } \\
\text { Levels Treated }\end{array}$ & $\%$ \\
\hline Total & $14 / 557$ & 2.5 & & $14 / 494$ & 2.8 \\
\hline Spinal level & & & & \\
\hline C-1 & $0 / 12$ & 0 & & $0 / 11$ & 0 \\
\hline C-2 & $0 / 18$ & 0 & & $0 / 17$ & 0 \\
\hline C-3 & $2 / 19$ & 10.5 & & $2 / 18$ & 11.1 \\
\hline C-4 & $0 / 11$ & 0 & & $0 / 10$ & 0 \\
\hline C-5 & $0 / 8$ & 0 & & $0 / 8$ & 0 \\
\hline C-6 & $1 / 15$ & 6.7 & & $1 / 12$ & 8.3 \\
\hline C-7 & $1 / 13$ & 7.7 & & $1 / 12$ & 8.3 \\
\hline T-1 & $1 / 19$ & 5.3 & & $1 / 15$ & 6.7 \\
\hline T2-11 & $0 / 250$ & 0 & & $0 / 219$ & 0 \\
\hline T-12 & $0 / 56$ & 0 & & $0 / 49$ & 0 \\
\hline L-1 & $1 / 51$ & 2.0 & & $1 / 44$ & 2.3 \\
\hline L-2 & $0 / 56$ & 0 & & $0 / 47$ & 0 \\
\hline L-3 & $2 / 52$ & 3.8 & $2 / 52$ & 3.8 \\
\hline L-4 & $1 / 36$ & 2.8 & $1 / 36$ & 2.8 \\
\hline L4-5 & $1 / 27$ & 3.7 & $1 / 23$ & 4.3 \\
\hline Sacrum & $4 / 47$ & 8.5 & $4 / 39$ & 10.2 \\
\hline
\end{tabular}




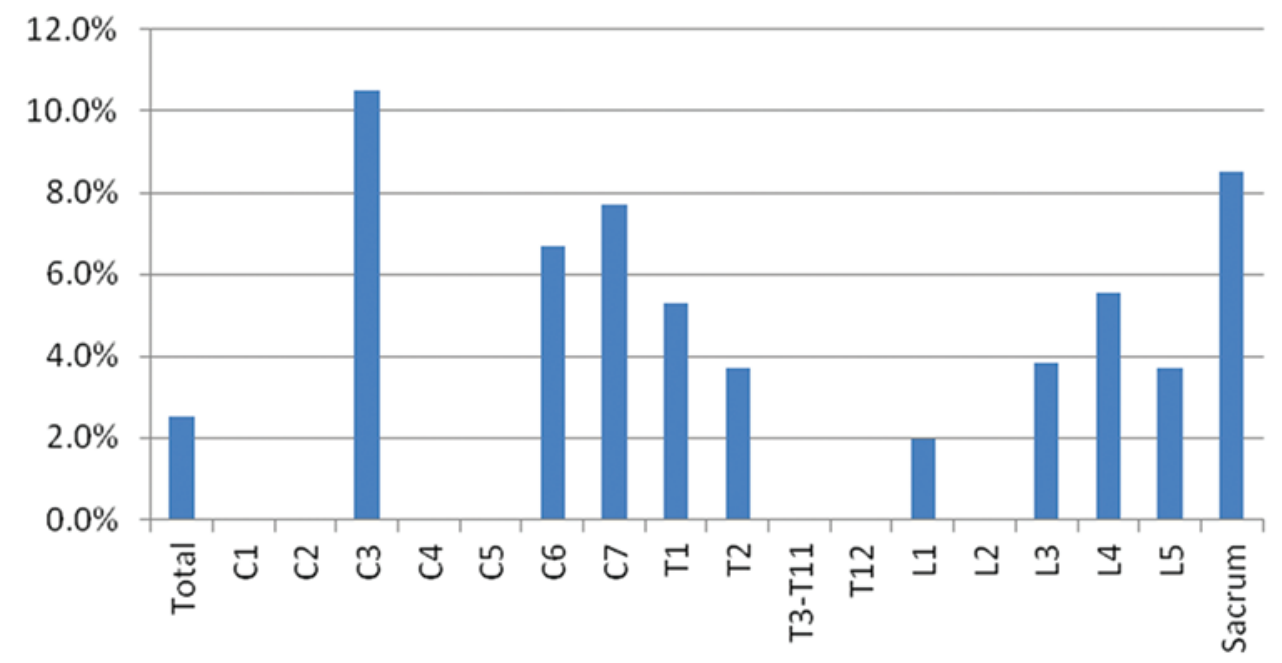

FIG. 1. Percentage of PNS injuries in all patients treated with SF-SRS (18-26 Gy) by spinal level.

those who did and those who did not develop neuropathy, whether it was the dose to 95\% of the GTV (GTV D95), the dose to $95 \%$ of the PTV (PTV D95), or the highest 5\% dose level given to the intended target (PTV D05). The mean GTV D95, PTV D95, and PTV D05 for patients who experienced neuropathy were 2416, 2294, and 2610 cGy, respectively. The mean GTV D95, PTV D95, and PTV D05 in 381 corresponding treatment plans for patients who did not experience neuropathy were 2353, 2205, and 2622 cGy, respectively. The target volume and treatment dose delivered do not explain the development of neuropathy. The overall percentage of PNS injuries for all high-dose SF-SRS treatments was $2.5 \%$. No PNS injuries were identified among the 306 cases in which treatment involved the thoracic spine (T2-12). Similarly, no PNS injuries were identified when treatment involved the C-1, C-2, C-4, C-5, T2-11, T-12, and L-2 segments. The highest percentage of PNS injury occurred when SF-SRS involved C-3 (2 [10.5\%] of 19 cases), followed by the sacrum (4 [8.5\%] of 47 cases), C-7 (1 [7.7\%] of 13 cases), C-6 (1 [6.7\%] of 15 cases), T-1 (1 [5.3\%] of 19 cases), L-3 (2 [3.8\%] of 52 cases), L4-5 (1 [3.7\%] of 27 cases), L-4 (1 [2.8\%] of 36 cases), and L-1 (1 [2.0\%] of 51 cases). When the thoracic spine was excluded, the percentage of SF-SRS treatments resulting in PNS injury was $4.5 \%$.

For those patients who developed nerve injuries, the median time to development of the PNS injury was 10 months following SF-SRS (range 4-32 months). Figure 2 plots the cumulative incidence of PNS injury and death at 1,2 , and 3 years. The estimated incidence of nerve injury at 1 year was $1.8 \%$ (95\% CI $1.0 \%-3.3 \%)$, increasing to $2.6 \%(95 \%$ CI $1.5 \%-4.3 \%)$ at 3 years. While all PNS injuries occurred when SF-SRS was delivered at $2400 \mathrm{cGy}$, dose was not significantly associated with the incidence of nerve injury $(\mathrm{p}=0.18)$.

The characteristics of PNS injury resulting from SFSRS are summarized in Table 4. The primary indication for SF-SRS in all patients was local tumor control. Specific indications for treatments resulting in PNS injury were pain (6 [43\%] of 14 cases) followed by oligometastatic prostate cancer (3 [21\%] of 14 cases). The patient was on hormonal therapy near the time of SF-SRS in a small number of cases (4 [28\%] of 14 cases). One (7\%) of 14 patients was on a vascular endothelial growth factor (VEGF) inhibitor near the time of radiation, but none were on chemotherapy. All patients received systemic therapy during the course of their treatment. Asymptomatic but radiographically evident PNS compression at the segment of interest was assessed on pretreatment imaging and found to be present before SF-SRS in 3 (21\%) of 14 treatments resulting in PNS injury. Only PN injuries in the radiation field were included in the analysis. Recurrence of disease at the radiated spinal segment was not thought to be responsible for neuropathic symptoms in any patient based on interpretation of imaging.

Electromyography was used to confirm clinical diagnoses in $9(64 \%)$ of 14 cases. Myokymia was present in only 1 (11\%) of 9 EMGs. Peripheral nervous system injuries were bilateral in $8(57 \%)$ of 14 cases. The plexus (cervical, brachial, and/or lumbosacral) was affected clinically and/ or electrophysiologically in $12(86 \%)$ of 14 cases. Isolated nerve root injury resulted from $2(14 \%)$ of 14 treatments. In both instances, radiation involved the upper lumbar spine. Radiculoplexopathy was evident in 6 (43\%) of 14 cases.

Neuropathic pain referable to PNS injury was present in all cases and weakness occurred in 13 (93\%) of 14. The only patient who did not demonstrate clinical weakness had irradiation at C-3 and suffered cervical plexopathy. Thirteen (93\%) of 14 PNS injuries required both pain management and physical/occupational therapies to optimize functional outcomes. Four (40\%) of 10 patients treated at the lumbosacral spine required an orthosis (ankle-foot orthosis or knee extension support brace) to compensate for strength deficits and facilitate ambulation; 2 (20\%) of the 10 required an assistive device such as a cane or walker. Two (14\%) of 14 treatments resulting in PNS injury were associated with lymphedema. Radiculopathy and/or plexopathy was CTCAE Grade 1 in $14 \%$ of cases, 2 in $64 \%$, and 3 in $21 \%$. Signs and symptoms including pain, weakness, and gait dysfunction worsened over time in 12 (71\%) of 14 cases. 


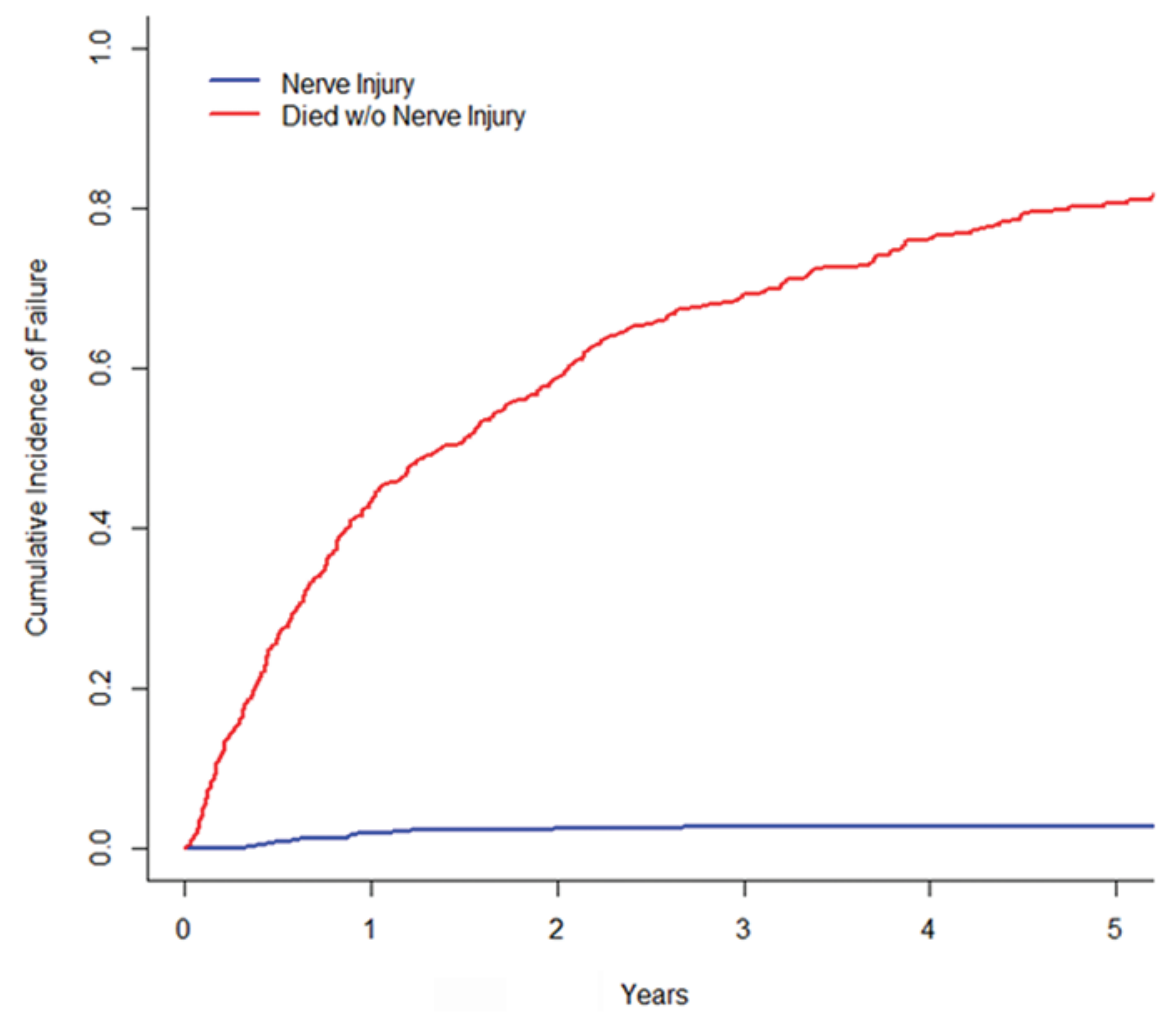

\begin{tabular}{|l|l|l|l|}
\hline & 1 year & 2 year & 3 year \\
\hline $\begin{array}{l}\text { Incidence of PNS injury } \\
\text { estimate }(95 \% \mathrm{Cl})\end{array}$ & $1.8 \%(1.0 \%-3.3 \%)$ & $2.4 \%(1.4 \%-4.1 \%)$ & $2.6 \%(1.5 \%-4.3 \%)$ \\
\hline $\begin{array}{l}\text { Incidence of death } \\
\text { estimate }(95 \% \mathrm{Cl})\end{array}$ & $43.3 \%(39.4 \%-47.7 \%)$ & $58.8 \%(54.8 \%-63.1 \%)$ & $69.2 \%(65.4 \%-73.3 \%)$ \\
\hline
\end{tabular}

FIG. 2. Time to PNS injury (in years since SF-SRS) using competing risk methods-18 to 26 Gy (557 treatments).

\section{Discussion}

The pathophysiology of radiation-induced PNS dysfunction is poorly understood. Damage to the local microvascular system is thought to result from perturbation of various cellular and molecular mechanisms with subsequent tissue fibrosis and atrophy. ${ }^{30}$ Risk factors for radiation-induced PNS injury include total dose, dose per fraction, volume of radiation, tissue type, prior radiation, local surgery, concomitant neurotoxic chemotherapy, and patient-related factors such as physiological status, comorbidities, preexisting PNS dysfunction, and genetic susceptibility. ${ }^{30}$

The reported risk of radiation-induced brachial plexopathy from conventionally fractionated radiotherapy (that is, $5000 \mathrm{cGy}$ in $200-\mathrm{Gy}$ fractions) is $<1 \%$. When the dose per fraction increases to between 2200 and 4580 cGy with a total dose of 4350 and $6000 \mathrm{~Gy}$, the risk of brachial plexus injury increases to $1.7 \%-73 \%{ }^{8}$ Similar dose-injury responses to PNS injury have been seen in the treatment of other cancer types and in animal models. 7,17,20

The overall percentage of PNS injury following SFSRS to the spine in our cohort was $2.5 \%$. This number increased when the thoracic spine was excluded. Signs and symptoms of PNS injury in the thoracic spine may be more difficult to detect clinically, as weakness is typically absent. Given that most referrals to the Rehabilitation Medicine Service were based on functional deficits and neuromuscular and musculoskeletal dysfunction as well as pain, it is possible that patients with symptomatic thoracic radiculopathy were not captured in our search and were instead treated for pain by their primary oncologist or radiation oncologist.

The percentage of treatments resulting in symptomatic PNS injury was $4.5 \%$ when the thoracic spine segments were excluded. This percentage compares well to other clinical contexts in which SF-SRS involves PNS structures. For instance, the rate of optic mononeuropathy is $5 \%$ in craniopharyngioma patients treated with SF-SRS at $>1500$ cGy and $10 \%$ following SF-SRS for skull base tumors treated at doses $>1200 \mathrm{cGy} .14,23$

As with other radiation delivery schema, PNS injury in SF-SRS has been found to be dose dependent with injury more likely at higher doses. ${ }^{20,21}$ In the present study, however, there was no significant dose response for PNS injury ( $p=0.18)$, even though no injuries resulting from SF-SRS delivered at doses $<2400 \mathrm{cGy}$ were identified. It should be noted that the number of PNS injury events and number of SF-SRS treatments at doses < 2400 cGy (63 [11\%] of 
TABLE 4. Peripheral nervous system injury resulting from SF-SRS

\begin{tabular}{|c|c|c|c|c|c|c|}
\hline $\begin{array}{l}\text { PNS } \\
\text { Injury }\end{array}$ & $\begin{array}{l}\text { Age } \\
\text { (yrs) }\end{array}$ & Sex & Target & PNS Structure(s) Affected & $\begin{array}{l}\text { Symptom } \\
\text { Onset (mos) }\end{array}$ & $\begin{array}{l}\text { CTCAE } \\
\text { Grade }\end{array}$ \\
\hline 1 & 47 & M & C-3 & Lt cervical plexopathy & 24 & 2 \\
\hline 2 & 45 & M & C-3 & Bilat cervical plexopathy & 10 & 1 \\
\hline 3 & 60 & M & C-6 & Bilat C5-7 cervical \& posterior cord brachial radiculoplexopathy & 32 & 2 \\
\hline 4 & 50 & M & C-7 & $\begin{array}{l}\text { Rt C7-8 radiculopathy \& middle/lower trunk brachial plexopathy, } \\
\text { It C-7 radiculopathy }\end{array}$ & 11 & 3 \\
\hline 5 & 48 & M & $\mathrm{T} 1-2$ & Bilat C8-T1 radiculopathy \& lower trunk brachial plexopathy & 7 & 2 \\
\hline 6 & 45 & M & L-1 & Lt L-1 radiculopathy & 10 & 1 \\
\hline 7 & 62 & M & $L-3$ & Bilat L2-4 radiculopathy & 6 & 2 \\
\hline 8 & 59 & M & L-3 & Lt L2-5 lumbosacral radiculoplexopathy, rt L2-4 radiculopathy & 13 & 2 \\
\hline 9 & 55 & M & L-4 & Bilat lumbosacral polyradiculoplexopathy & 4 & 2 \\
\hline 10 & 63 & $\mathrm{~F}$ & L-4, L-5 & $\begin{array}{l}\text { Rt L2-S1 lumbosacral radiculoplexopathy, It L5-S1 lumbosacral } \\
\text { radiculopathy }\end{array}$ & 7 & 3 \\
\hline 11 & 71 & M & SI joint & Lt lumbosacral plexopathy & 4 & 2 \\
\hline 12 & 66 & $\mathrm{~F}$ & Sacrum & Lt lumbosacral plexopathy & 11 & 3 \\
\hline 13 & 31 & $\mathrm{~F}$ & Sacrum & Lt lumbosacral plexopathy & 14 & 2 \\
\hline 14 & 60 & $\mathrm{~F}$ & Rt sacrum & Rt lumbosacral plexopathy & 5 & 2 \\
\hline
\end{tabular}

overall cohort) were small, so the study may have lacked adequate power to detect a dose response.

All the nerve injuries in this cohort involved the nerve root and/or plexus as assessed clinically and/or electrophysiologically. This was expected given the anatomical location of the nerve root and plexus within the treatment field of SF-SRS. The pattern of motor, sensory, and reflex deficits helps to differentiate radiculopathy from plexopathy clinically. A key feature separating plexopathy from radiculopathy electrophysiologically is the abnormal sensory responses in the affected dermatome in the absence of a generalized peripheral neuropathy. In radiculopathy the dorsal root ganglion (DRG) is spared and thus sensory testing on nerve conduction studies is normal. Therefore, the DRG serves as the anatomical demarcation separating nerve root from plexus. Needle EMG is critical in differentiating radiculopathy from plexopathy but is challenging given that key muscles such as the paraspinal muscles, which are often abnormal in radiculopathy but not plexopathy, can be damaged by direct radiation effects and thus confound diagnosis.

Historically, myokymia has been present in approximately $60 \%$ of patients with radiation-induced plexopathy. While the association of myokymia with conventional radiation is well known, there are no data on the incidence of myokymia resulting from SF-SRS to the spine. In our cohort, myokymia was present in only 1 (11\%) of 9 cases with EMG studies, suggesting that the condition may be less common in patients treated with SF-SRS than those treated with conventional radiotherapy.

In the patients with PNS injuries, the median time to symptom onset was 10 months following SF-SRS (range 4-32 months). At 1 year after treatment, only 1.8\% (95\% CI $1.0 \%-3.3 \%$ ) of the patients were estimated to have PNS injury, but $43.3 \%$ (95\% CI 39.4\%-47.7\%) were estimated to have died. By 3 years following SF-SRS, the PNS injury estimate increased to $2.6 \%$ (95\% CI $1.5 \%-4.3 \%$ ) and the estimated incidence of death increased to $69.2 \%$ (95\% CI $65.4 \%-73.3 \%$ ). Because SF-SRS is generally administered with palliative as opposed to curative intent, it is possible that patients who may have developed PNS injury died before the injury could manifest.

The clinical sequelae of PNS injury in our cohort were often significant (64\% CTCAE Grade 2, 21\% Grade 3) with major implications for function and quality of life. All of the patients had pain related to their PNS injury, and all but one, a patient with cervical plexopathy, had weakness. The majority of injuries (93\%) required pain management and physical and/or occupational therapy. Additionally, $40 \%$ of the patients treated at the lumbosacral spine required an orthotic device for ambulation and $20 \%$ required an assistive device such as a cane or walker. Signs and symptoms continued to worsen in most patients but stabilized in 2 cases.

The clinical presentation of PNS injury following highdose SF-SRS to the spine in our cohort differs from what is traditionally anticipated. The onset of symptoms in our cohort may be earlier than what is generally reported for conventionally fractionated radiation, and all of the patients had pain, which is not considered typical. ${ }^{17}$ While radiation plexopathy is often described as a painless loss of function, pain can be a feature in approximately $20 \%$ of cases resulting from traditionally fractionated radiation therapy. ${ }^{21}$ Pain resulting from radiation damage to nerve roots has not been well described. The majority of our patients had damage to both the nerve root and the plexus, which may have contributed to the high incidence of pain. Additionally, the enhanced biological effects of high-dose SF-SRS on both tumor and normal tissues may have contributed to differences in presentation through accelerated vascular compromise of the affected neural structures. ${ }^{35}$

Radiotoxicity may be enhanced by a variety of new, 
targeted antineoplastic agents such the EGFR and VEGF inhibitors. ${ }^{11,29}$ Additionally, premorbid degenerative spine disease, prior exposure to neurotoxic chemotherapy, and other causes of nerve dysfunction may predispose to PNS dysfunction in the setting of radiotherapy. As new oncological treatments become available, practitioners should be vigilant regarding their potential to adversely affect the PNS when such treatments are combined with radiation therapy.

The major limitations of this study include its retrospective design and lack of uniformity in identifying patients with PNS injury. Patients with such injuries were identified because they had been referred to the Rehabilitation Medicine Service, thus introducing sample bias. Patients with clinically less severe injuries treated by their oncologists may not have been identified; therefore, the number of injuries was underestimated and assessment of their severity was affected. Certain predisposing medical conditions such as diabetes are known to cause peripheral neuropathy. In the current study, however, these conditions were not taken into account for contouring and dosimetry purposes and could be interesting to further prospective investigations. Additionally, direct neoplastic spread through the peripheral nerves (for example, breast cancer spread to the brachial plexus ${ }^{15,37}$ ) has been described and was not routinely addressed herein with PET and/or CT. Prospective study would benefit from uniform clinical, electrophysiological, pain, and quality of life assessment.

\section{Conclusions}

Single-fraction SRS has emerged as an important modality in the treatment of both primary and metastatic spine tumors. While this technology may offer benefits relative to those of conventional radiotherapy, it is not without risk. Peripheral nervous system injury occurs in $4.2 \%$ of cases when high-dose SF-SRS is delivered to the cervical or lumbosacral spine. Though such injuries are limiting in terms of function and quality of life in affected patients, this percentage is similar, if not better, than the documented incidence of PNS injury when using other radiation techniques. As with all cancer treatments, the potential risks must be weighed against potential benefits.

\section{Acknowledgments}

This research was funded in part through the NIH/NCI Cancer Center Support Grant No. P30 CA008748.

\section{References}

1. Balagamwala EH, Angelov L, Koyfman SA, Suh JH, Reddy CA, Djemil T, et al: Single-fraction stereotactic body radiotherapy for spinal metastases from renal cell carcinoma. J Neurosurg Spine 17:556-564, 2012

2. Collen C, Ampe B, Gevaert T, Moens M, Linthout N, De Ridder M, et al: Single fraction versus fractionated linacbased stereotactic radiotherapy for vestibular schwannoma: a single-institution experience. Int J Radiat Oncol Biol Phys 81:e503-e509, 2011

3. Cox BW, Jackson A, Hunt M, Bilsky M, Yamada Y: Esophageal toxicity from high-dose, single-fraction paraspinal stereotactic radiosurgery. Int J Radiat Oncol Biol Phys 83:e661-e667, 2012
4. Cox BW, Spratt DE, Lovelock M, Bilsky MH, Lis E, Ryu S, et al: International Spine Radiosurgery Consortium consensus guidelines for target volume definition in spinal stereotactic radiosurgery. Int J Radiat Oncol Biol Phys 83:e597e605, 2012

5. Dueck AC, Mendoza TR, Mitchell SA, Reeve BB, Castro KM, Rogak LJ, et al: Validity and reliability of the US National Cancer Institute's Patient-Reported Outcomes version of the Common Terminology Criteria for Adverse Events (PRO-CTCAE). JAMA Oncol 1:1051-1059, 2015

6. Folkert MR, Bilsky MH, Tom AK, Oh JH, Alektiar KM, Laufer I, et al: Outcomes and toxicity for hypofractionated and single-fraction image-guided stereotactic radiosurgery for sarcomas metastasizing to the spine. Int J Radiat Oncol Biol Phys 88:1085-1091, 2014

7. Forquer JA, Fakiris AJ, Timmerman RD, Lo SS, Perkins SM, McGarry RC, et al: Brachial plexopathy from stereotactic body radiotherapy in early-stage NSCLC: dose-limiting toxicity in apical tumor sites. Radiother Oncol 93:408-413, 2009

8. Gałecki J, Hicer-Grzenkowicz J, Grudzień-Kowalska M, Michalska T, Załucki W: Radiation-induced brachial plexopathy and hypofractionated regimens in adjuvant irradiation of patients with breast cancer-a review. Acta Oncol 45:280-284, 2006

9. Garg AK, Shiu AS, Yang J, Wang XS, Allen P, Brown BW, et al: Phase $1 / 2$ trial of single-session stereotactic body radiotherapy for previously unirradiated spinal metastases. Cancer 118:5069-5077, 2012

10. Gerszten PC, Burton SA, Ozhasoglu C, Vogel WJ, Welch WC, Baar J, et al: Stereotactic radiosurgery for spinal metastases from renal cell carcinoma. J Neurosurg Spine 3:288295,2005

11. Gibbs IC, Kamnerdsupaphon P, Ryu MR, Dodd R, Kiernan $M$, Chang SD, et al: Image-guided robotic radiosurgery for spinal metastases. Radiother Oncol 82:185-190, 2007

12. Gibbs IC, Patil C, Gerszten PC, Adler JR Jr, Burton SA: Delayed radiation-induced myelopathy after spinal radiosurgery. Neurosurgery 64 (2 Suppl):A67-A72, 2009

13. Gray RJ: A class of K-sample tests for comparing the cumulative incidence of a competing risk. Ann Stat 16:1141-1154, 1988

14. Hasegawa T, Kobayashi T, Kida Y: Tolerance of the optic apparatus in single-fraction irradiation using stereotactic radiosurgery: evaluation in 100 patients with craniopharyngioma. Neurosurgery 66:688-695, 2010

15. Ho L, Henderson R, Luong T, Malkhassian S, Wassef H: ${ }^{18} \mathrm{~F}-$ FDG PET/CT appearance of metastatic brachial plexopathy involving epidural space from breast carcinoma. Clin Nucl Med 37:e263-e264, 2012

16. Howell DD, James JL, Hartsell WF, Suntharalingam M, Machtay M, Suh JH, et al: Single-fraction radiotherapy versus multifraction radiotherapy for palliation of painful vertebral bone metastases-equivalent efficacy, less toxicity, more convenient: a subset analysis of Radiation Therapy Oncology Group trial 97-14. Cancer 119:888-896, 2013

17. Jaeckle KA: Neurologic manifestations of neoplastic and radiation-induced plexopathies. Semin Neurol 30:254-262, 2010

18. Kalbfleisch JD, Prentice RL: The Statistical Analysis of Failure Time Data. New York: Wiley, 1980

19. Katagiri H, Takahashi M, Inagaki J, Kobayashi H, Sugiura $\mathrm{H}$, Yamamura $\mathrm{S}$, et al: Clinical results of nonsurgical treatment for spinal metastases. Int J Radiat Oncol Biol Phys 42:1127-1132, 1998

20. Kinsella TJ, Sindelar WF, DeLuca AM, Pezeshkpour G, Smith R, Maher M, et al: Tolerance of peripheral nerve to intraoperative radiotherapy (IORT): clinical and experimental studies. Int J Radiat Oncol Biol Phys 11:1579-1585, 1985 
21. Kori SH, Foley KM, Posner JB: Brachial plexus lesions in patients with cancer: 100 cases. Neurology 31:45-50, 1981

22. Laufer I, Iorgulescu JB, Chapman T, Lis E, Shi W, Zhang Z, et al: Local disease control for spinal metastases following "separation surgery" and adjuvant hypofractionated or highdose single-fraction stereotactic radiosurgery: outcome analysis in 186 patients. J Neurosurg Spine 18:207-214, 2013

23. Leavitt JA, Stafford SL, Link MJ, Pollock BE: Long-term evaluation of radiation-induced optic neuropathy after singlefraction stereotactic radiosurgery. Int J Radiat Oncol Biol Phys 87:524-527, 2013

24. Ling CC, Burman C, Chui CS, Kutcher GJ, Leibel SA, LoSasso T, et al: Conformal radiation treatment of prostate cancer using inversely-planned intensity-modulated photon beams produced with dynamic multileaf collimation. Int J Radiat Oncol Biol Phys 35:721-730, 1996

25. Lovelock DM, Hua C, Wang P, Hunt M, Fournier-Bidoz N, Yenice K, et al: Accurate setup of paraspinal patients using a noninvasive patient immobilization cradle and portal imaging. Med Phys 32:2606-2614, 2005

26. Martin AG, Cowley IR, Taylor BA, Cassoni AM, Landau DB, Plowman PN: (Stereotactic) radiosurgery XIX: spinal radiosurgery-two year experience in a UK centre. Br J Neurosurg 26:53-58, 2012

27. Mohan R, Barest G, Brewster LJ, Chui CS, Kutcher GJ, Laughlin JS, et al: A comprehensive three-dimensional radiation treatment planning system. Int J Radiat Oncol Biol Phys 15:481-495, 1988

28. Moulding HD, Elder JB, Lis E, Lovelock DM, Zhang Z, Yamada Y, et al: Local disease control after decompressive surgery and adjuvant high-dose single-fraction radiosurgery for spine metastases. J Neurosurg Spine 13:87-93, 2010

29. Niyazi M, Maihoefer C, Krause M, Rödel C, Budach W, Belka C: Radiotherapy and "new" drugs-new side effects? Radiat Oncol 6:177, 2011

30. Pradat PF, Delanian S: Late radiation injury to peripheral nerves. Handb Clin Neurol 115:743-758, 2013

31. Rades D, Huttenlocher S, Bajrovic A, Karstens JH, Adamietz IA, Kazic N, et al: Surgery followed by radiotherapy versus radiotherapy alone for metastatic spinal cord compression from unfavorable tumors. Int J Radiat Oncol Biol Phys 81:e861-e868, 2011

32. Rose PS, Laufer I, Boland PJ, Hanover A, Bilsky MH, Yamada J, et al: Risk of fracture after single fraction image-guided intensity-modulated radiation therapy to spinal metastases. J Clin Oncol 27:5075-5079, 2009
33. Sahgal A, Ma L, Gibbs I, Gerszten PC, Ryu S, Soltys S, et al: Spinal cord tolerance for stereotactic body radiotherapy. Int J Radiat Oncol Biol Phys 77:548-553, 2010

34. Sahgal A, Weinberg V, Ma L, Chang E, Chao S, Muacevic A, et al: Probabilities of radiation myelopathy specific to stereotactic body radiation therapy to guide safe practice. Int $\mathbf{J}$ Radiat Oncol Biol Phys 85:341-347, 2013

35. Yamada Y, Bilsky MH, Lovelock DM, Venkatraman ES, Toner S, Johnson J, et al: High-dose, single-fraction imageguided intensity-modulated radiotherapy for metastatic spinal lesions. Int J Radiat Oncol Biol Phys 71:484-490, 2008

36. Yamada Y, Laufer I, Cox BW, Lovelock DM, Maki RG, Zatcky JM, et al: Preliminary results of high-dose singlefraction radiotherapy for the management of chordomas of the spine and sacrum. Neurosurgery 73:673-680, 2013

37. Zingale A, Ponzo G, Ciavola G, Vagnoni G: Metastatic breast cancer delayed brachial plexopathy. A brief case report. J Neurosurg Sci 46:147-149, 2002

\section{Disclosures}

Dr. Laufer is a consultant for Depuy/Synthes, Globus, and SpineWave. Dr. Lis is a consultant for Medtronic. Dr. Yamada is a consultant for Varian Medical Systems and a medical advisory board member of the Chordoma Foundation. Dr. Bilsky is a consultant for Globus and BrainLab and receives royalties from Depuy/Synthes.

\section{Author Contributions}

Conception and design: Bilsky, Stubblefield, Laufer, Yamada. Acquisition of data: Stubblefield, Ibanez, Lis, Yamada. Analysis and interpretation of data: Bilsky, Stubblefield, Ibanez, Riedel, Laufer, Lis, Yamada. Drafting the article: Stubblefield. Critically revising the article: Bilsky, Stubblefield, Barzilai, Laufer. Reviewed submitted version of manuscript: all authors. Approved the final version of the manuscript on behalf of all authors: Bilsky. Statistical analysis: Stubblefield, Riedel. Administrative/ technical/material support: Stubblefield, Ibanez, Riedel, Barzilai. Study supervision: Bilsky, Stubblefield, Laufer.

\section{Correspondence}

Mark H. Bilsky, Department of Neurosurgery, Memorial Sloan Kettering Cancer Center, 1275 York Ave., c705, New York, NY 10021. email: bilskym@mskcc.org. 